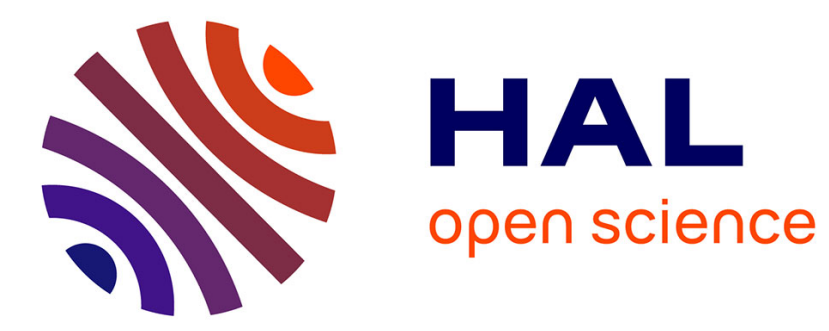

\title{
Design, manufacturing, and characterization of copper capillary structures for loop heat pipes
}

Remi Giraudon, Stéphane Lips, D. Fabrègue, Laurent Gremillard, E. Maire, Valérie Sartre

\section{- To cite this version:}

Remi Giraudon, Stéphane Lips, D. Fabrègue, Laurent Gremillard, E. Maire, et al.. Design, manufacturing, and characterization of copper capillary structures for loop heat pipes. Heat Pipe Science and Technology, An International Journal, 2017, 8 (1), pp.27 - 49. 10.1615/HeatPipeScieTech.2017018805 . hal-01735918

\section{HAL Id: hal-01735918 https://hal.science/hal-01735918}

Submitted on 20 Mar 2019

HAL is a multi-disciplinary open access archive for the deposit and dissemination of scientific research documents, whether they are published or not. The documents may come from teaching and research institutions in France or abroad, or from public or private research centers.
L'archive ouverte pluridisciplinaire HAL, est destinée au dépôt et à la diffusion de documents scientifiques de niveau recherche, publiés ou non, émanant des établissements d'enseignement et de recherche français ou étrangers, des laboratoires publics ou privés. 


\title{
Design, manufacturing and characterization of copper capillary structures for loop heat pipes
}

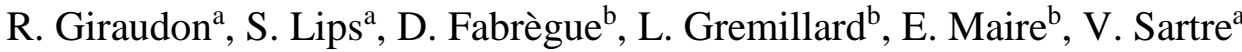 \\ ${ }^{a}$ Univ Lyon, CNRS, INSA-Lyon, CETHIL UMR5008, F-69621, Villeurbanne, France \\ ${ }^{b}$ Univ Lyon, CNRS, INSA-Lyon, MATEIS UMR5510, F-69621, Villeurbanne, France
}

\begin{abstract}
Mono and bi-layer capillary structures are designed by means of a thermo-hydraulic model of a loop heat pipe in order to optimize the performance of the system. The model tends to show that bi-layer wicks with a thermally conducting bottom layer and an insulating top layer are the most efficient in loop heat pipes. An experimental study is then led to manufacture and characterize the bottom layer. Eight samples made of copper powder are manufactured following a twolevel fractional factorial design. The top layer is not manufactured in this study. The sintering parameters are adjusted to provide porous samples with sufficient mechanical resistance. The porous structure permeability and its capacity to provide a sufficient capillary pressure are evaluated using a specific test bench dedicated to this study, as well as with microstructural observations (tomography, microscopy). The experimental characterization of the samples enables to determine the influence of each sintering parameter as well as the interactions between them. The characteristics of the porous samples are found to be mainly affected by the sintering time and the pressure. High values of these parameters decrease the permeability and the porosity but increase the maximum capillary pressure due to a smaller effective pore radius. A set of optimum sintering parameters is found in order to manufacture the bottom layer. The best porous structure is supposed to enhance the latent heat transfer in a LHP.
\end{abstract}

Keywords: loop heat pipe; capillary structure; porosity; permeability; pore radius; manufacturing; sintering

\section{INTRODUCTION}

The increase of the heat flux densities encountered in industrial applications and the decrease of the electronic components size require efficient cooling devices to avoid failure due to thermal issues. Among them, the passive systems that use the liquid-vapour phase change have many advantages. They don't require additional energy source and can transport high heat flux densities. Loop heat pipes (LHPs) proved their ability to cool systems in a large temperature range. Ku (1999) and Maydanik (2005) deeply explained the LHP operating principles and the components characteristics. Contrarily to traditional heat pipes, the capillary structure is located only in the evaporator. Confining the wick in the evaporator section enhances the capillary limit as explained by Singh et al. (2014). The wick is a key element of the LHP and many authors investigated it. Demidov and Yatsenko 
(1994) as well as Cao and Faghri (1994) studied numerically its operation whereas Zhao and Liao (2000) and Mottet et al. (2015) proposed experimental studies. According to Launay and Vallée (2011), the large majority of LHPs is designed with a monoporous, monolayer and metallic wick. However, this technology has shown its limits and the challenge to increase the LHP performances is to find out well-adapted capillary structures to enhance the heat and mass transfer in the evaporator. The collaboration between material scientists and heat transfer researchers can help to select the most appropriate fabrication method for the considered application. Some scientists started this kind of work and tried to manufacture new types of wick. Yeh et al. (2008) studied a biporous wick. The biporous wick was manufactured by mixing a $3 \mu \mathrm{m}$ nickel powder and a pore former. The biporous wick decreased the thermal resistance from $0.53 \mathrm{~K} / \mathrm{W}$ to $0.31 \mathrm{~K} / \mathrm{W}$ compared to a monoporous wick manufactured without pore former. They used a two-level fractional design to show that the pore former content was the most influent parameter. The particle size of pore former was influent but to a less extent and the sintering temperature was the less important parameter. Wu et al. (2013) studied the double layer wick. The first layer was biporous in order to increase the permeability while the second layer was monoporous in order to increase the mechanical strength of the whole wick. They sintered a nickel powder with a diameter lying between $2.2 \mu \mathrm{m}$ and $2.8 \mu \mathrm{m}$ and added a large pore former (particle diameter between $177 \mu \mathrm{m}$ and $210 \mu \mathrm{m}$ ) to create the biporous wick. They exhibited a positive influence on the LHP thermal performance. Indeed, the maximum heat flux was increased by $67 \%$ compared to mono layer and mono porous wicks $\left(11,6 \mathrm{~W} / \mathrm{cm}^{2}\right.$ compared to $\left.6.96 \mathrm{~W} / \mathrm{cm}^{2}\right)$. $\mathrm{Xu}$ et al. (2014) tested a multi-layer and bi-porous wick. The layer close to the evaporator had a high thermal conductivity in order to transfer the heat to the liquid and was biporous to evacuate more efficiently the vapour. The second layer had also a high thermal conductivity in order to spread the heat and was monoporous to create a better capillary force and finally the layer close to the reservoir was insulating to create a thermal barrier between the evaporator and the reservoir. Their results showed that these wicks improve the performance of their LHP. Indeed, they reported a significant decrease of the evaporator temperature and a maximum heat flux density of $40 \mathrm{~W} / \mathrm{cm}^{2}$. However, no modelling was proposed in order to find the best characteristics of each layers.

Based on these considerations, the main objective of this work is to determine, by means of a model, the most appropriate porous structure characteristics in order to provide high heat transfer performance of the LHP. Mono and bi-layer capillary structures are considered. The second objective is to manufacture capillary structures having similar characteristics using a sintering method. In this study, the performance of a bi-layer wick is investigated by means of simulations but only the layer in contact with the evaporator is manufactured. Indeed, since the heat transfer takes place inside this layer, it is of great interest to control its parameters. Finally, the hydrodynamic parameters of the 
manufactured samples are characterized and the influence of the parameters of the sintering process on the wick characteristics are discussed.

\section{WICK DESIGN}

The first step of this work is to develop a complete LHP model with a bi-layer wick. The goal is to determine the best wick characteristics, by means of a parametric analysis on each parameter of the wick: porosity $\varepsilon$, pore radius $r_{p}$, permeability $K$, thermal conductivity $\lambda$ and thickness $e$.

\subsection{Thermal and hydrodynamic model}

The modelled LHP is expected to cool an electronic component dissipating a heat flux up to $10 \mathrm{~W} / \mathrm{cm}^{2}$. Siedel et al. (2015) developed a model to predict the thermal and hydrodynamic behaviors of a flat-disk shaped LHP. The objective of his model is to find evaporator designs enabling to reduce the heat leaks from the heating plate to the reservoir, while shortening the thermal path between the heating plate and the evaporating interface. The model of Siedel et al. (2015) considers a monolayer nickel wick saturated with liquid. In the present study, it is modified to consider the influence of a double layer wick with different characteristics at the bottom part (in contact with the heated wall) and at the top part (in contact with the reservoir) (Fig. 1).
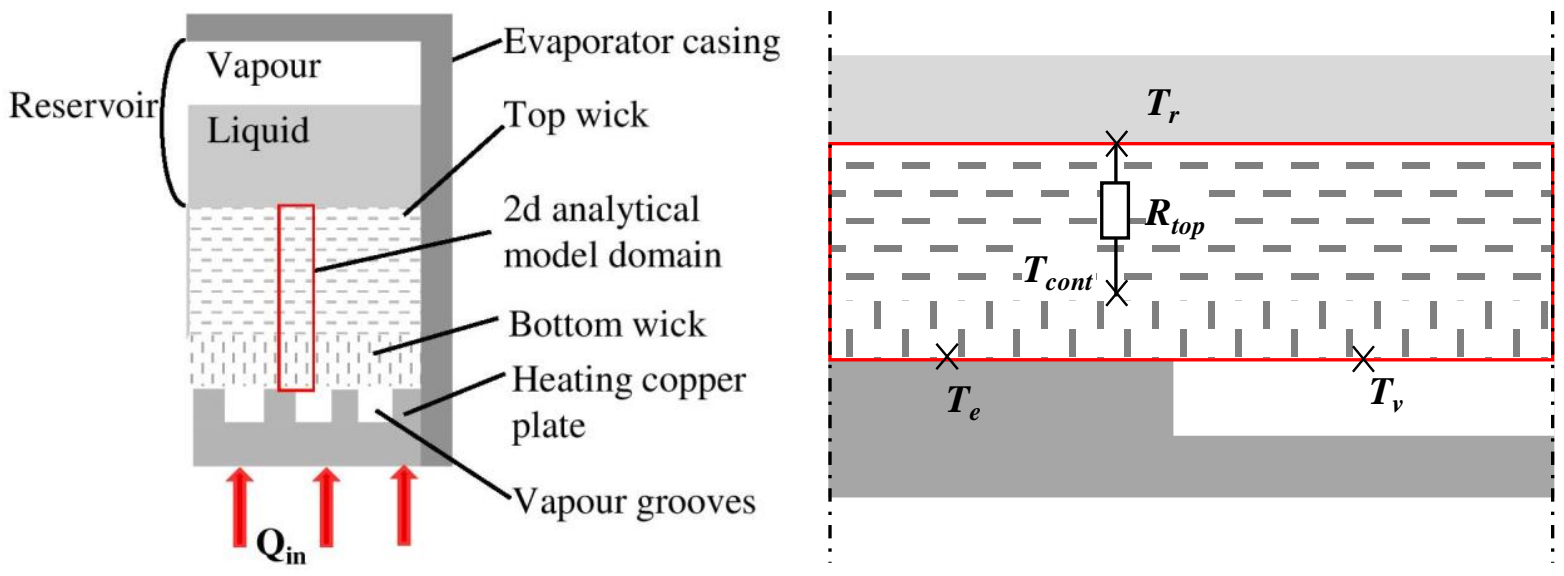

Fig. 1 : Schematic of the evaporator with double layer wick

The model of Siedel et al. (2015) is a nodal network based on a combination of energy balance equations for each component of the LHP. To precisely describe the heat and mass transfer inside evaporator casing / porous wick assembly, two analytical models were developed and coupled with the nodal model. In the first one, a small element located at the evaporator edge is considered. In this zone, the heat exchanged from the evaporator casing to the ambient (heat flux leaking from the wall) 
is influent. In the second analytical model, a small element is considered at the evaporator center, constituted by half a groove and half a fin (Fig. 1). This zone is not affected by the boundary effects, and the model enables to determine the heat and mass transfer inside the wick (heat flux dissipated by evaporation and leaking through the wick). The equations of the nodal network of Siedel et al. (2015) are presented in the Table 1, column 1 . The $k_{n}$ coefficients are determined are calculated by solving the analytical models. The equations of the model are solved by means of a MATLAB code.

Table 1 : Equations of the Siedel et al. (2015) model and modifications

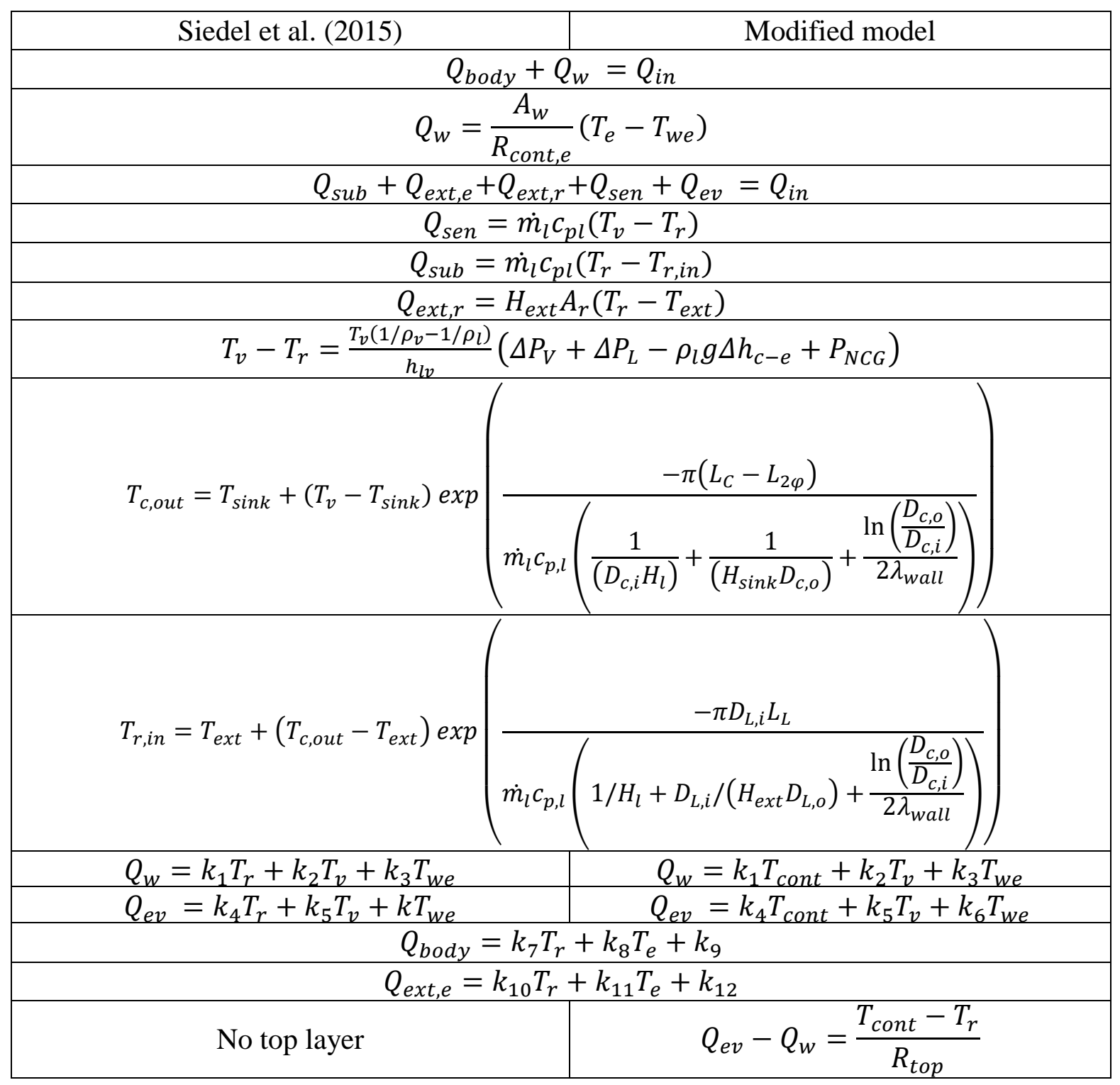

The simulations performed on the monolayer wick showed that the isotherms distortion only occurs in a very thin layer at the vicinity of the heated wall. Thus, when modifying the model to consider a bi-layer wick, a 1D heat transfer can be considered in the top layer, by adding a thermal resistance $R_{\text {top }}$ between the bottom wick and the liquid in the reservoir (Table 1, column 2). $R_{\text {top }}$ represents a conductive thermal resistance inside the top layer combined with the transport of the working fluid 
through the wick, resulting from the integration of a 1D heat and mass balance on a wick slice (Launay et al., 2008):

$$
R_{\text {top }}=\frac{\exp \left(\frac{\dot{m}_{l} c_{p, l}}{\lambda_{e f f} A_{w}} e_{t o p}\right)-1}{\dot{m}_{l} c_{p, l}}
$$

where $\dot{m}$ is the working fluid mass flow rate, $c_{p}$ the liquid specific heat, $e_{t o p}$ the top layer thickness, $\lambda_{\text {eff }}$ the top layer effective thermal conductivity and $A_{w}$ the wick cross-sectional area.

The imposed boundary conditions are the temperature of the condenser, the ambient temperature and the heat flux. The model enables to determine the temperature of each node and particularly the reservoir temperature $T_{r}$, the heating plate temperature $T_{e}$ and the vapour temperature $T_{v}$. The temperature at the interface of the two layers $T_{\text {cont }}$ is assumed to be constant along the surface. An example of the temperature field obtained with the LHP geometrical parameters and the operating conditions listed in Table 2 is presented in Fig. 2. The signification of each parameter is detailed in (Siedel et al., 2015).

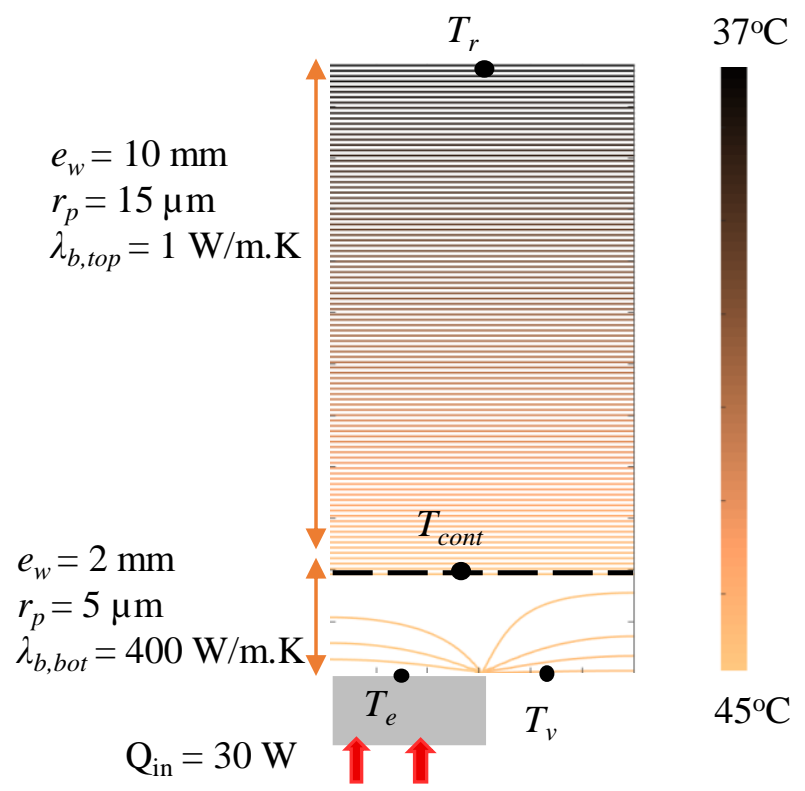

Fig. 2: Detail of the 2D modelled domain with the temperature field for the conditions listed in Table 2

Table 2 : LHP geometrics parameters

\begin{tabular}{|c|c|c|c|c|c|}
\hline \multicolumn{2}{|c|}{ Operating conditions } & \multicolumn{2}{c|}{$\begin{array}{c}\text { Wick parameters (top } \\
\text { layer) }\end{array}$} & \multicolumn{2}{c|}{ Other parameters } \\
\hline$Q_{\text {in }}$ & $30 \mathrm{~W}$ & $r_{p, \text { top }}$ & $15 \mu \mathrm{m}$ & $H_{\text {sink }}$ & $600 \mathrm{~W} / \mathrm{m}^{2} . \mathrm{K}$ \\
\hline$T_{\text {sink }}$ & $15^{\circ} \mathrm{C}$ & $\varepsilon_{t o p}$ & 0.45 & $a_{e v}$ & 0.6 \\
\hline$T_{a m b}$ & $25^{\circ} \mathrm{C}$ & $e_{t o p}$ & $10 \mathrm{~mm}$ & $P_{N C G}$ & $0 \mathrm{~Pa}$ \\
\hline
\end{tabular}




\begin{tabular}{|c|c|c|c|c|c|}
\hline Fluid & water & $\lambda_{b, t o p}$ & $1 \mathrm{~W} / \mathrm{m} . \mathrm{K}$ & $D_{e}$ & $40 \mathrm{~mm}$ \\
\hline Position & horizontal & $K_{\text {top }}$ & $2.210^{-12} \mathrm{~m}^{2}$ & $h_{r}$ & $20 \mathrm{~mm}$ \\
\hline \multirow{2}{*}{ Transport line parameters } & \multicolumn{2}{|c|}{$\begin{array}{r}\text { Wick parameters } \\
\text { (bottom layer) }\end{array}$} & $e_{\text {body }}$ & $3 \mathrm{~mm}$ \\
\hline$L_{V}$ & $685 \mathrm{~mm}$ & $r_{p, \text { bot }}$ & $5 \mu \mathrm{m}$ & $\lambda_{\text {body }}$ & $0.25 \mathrm{~W} / \mathrm{m} . \mathrm{K}$ \\
\hline$L_{c}$ & $250 \mathrm{~mm}$ & $\varepsilon_{b o t}$ & 0.45 & $R_{\text {cont,e }}$ & $3.10^{-4} \mathrm{Km}^{2} / \mathrm{W}$ \\
\hline$L_{L}$ & $245 \mathrm{~mm}$ & $e_{b o t}$ & $2 \mathrm{~mm}$ & $L_{g r}$ & $2 \mathrm{~mm}$ \\
\hline$\lambda_{\text {wall }}$ & $1 \mathrm{~W} / \mathrm{m} . \mathrm{K}$ & $\lambda_{b, b o t}$ & $400 \mathrm{~W} / \mathrm{m} . \mathrm{K}$ & & \\
\hline$D_{i^{-}} D_{o}$ & $2-6 \mathrm{~mm}$ & $K_{b o t}$ & $2.510^{-13} \mathrm{~m}^{2}$ & & \\
\hline
\end{tabular}

A sensitivity study is performed in the two following sections of this work. It is conducted on the parameters of each wick layer, namely the thickness, the porosity, the permeability, the pore radius and the thermal conductivity. In each simulation, only one parameter varies, the others being fixed at a reference value given in Table 2 .

\subsection{Thermal behaviour considerations}

The LHP thermal behaviour is studied by observing the influence of various parameters on the reservoir temperature and the boiling limit. A low reservoir temperature indicates a good thermal performance of the loop. Indeed, it means that the condenser thermal resistance is low and that the heat leaking to the reservoir is limited. The effect of the top layer thickness and of its material are studied by means of the model, in order to understand the influence of these parameters. The material is characterized by its bulk thermal conductivity which is linked to the effective thermal conductivity of the porous medium thanks to the Alexander's correlation (Alexander, 1972):

$$
\lambda_{e f f}=\lambda_{l}\left(\frac{\lambda_{b}}{\lambda_{l}}\right)^{(1-\varepsilon)^{0,59}}
$$

where $\lambda_{l}$ and $\lambda_{b}$ are the thermal conductivities of the liquid and the wick bulk material, respectively. Fig. 3 shows that, for a constant bottom layer thickness, increasing the thickness of the top layer decreases the reservoir temperature. However, this decrease is not significant beyond $10 \mathrm{~mm}$. Fig. 3 also shows that the top layer material is influent. An increasing thermal conductivity increases the reservoir temperature. This phenomenon is particularly true if the thermal conductivity is higher than $0.1 \mathrm{~W} / \mathrm{m}$.K. It means that the thermal conductivity has to be kept below this value if possible. Plastics, polymers and ceramics can be used to manufacture the top layer since their thermal conductivity is low. This conclusion is consistent with the experimental works of Boo and Chung (2005), who 
showed that it was possible to use polypropylene in LHPs, and Santos et al. (2010) who used ceramics for the same purpose.

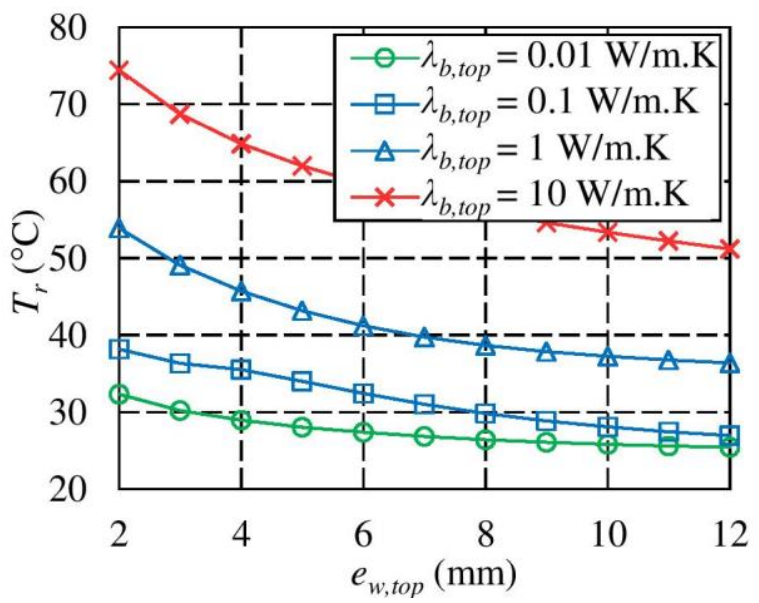

Fig. 3: Effect of the top layer properties on the temperature reservoir

The same study is realized concerning the bottom layer. Fig. 4 shows that, for a fixed top layer thickness, the bottom layer thickness has almost no influence on the reservoir temperature. Thus, the thickness must be reduce as much as possible in order to decrease the pressure losses through the porous wick while keeping a sufficient mechanical strength. The thermal conductivity of the bottom layer material has a small influence, especially when it is higher than $10 \mathrm{~W} / \mathrm{m} . \mathrm{K}$. As a consequence, the bottom layer thermal conductivity must be higher than this value in order to enhance the heat transfer between the evaporator wall and the menisci where the evaporation takes place. Metals can be used to manufacture the bottom layer since their thermal conductivity is high. For instance, Launay and Vallée (2011) have shown that copper and nickel are good candidates.

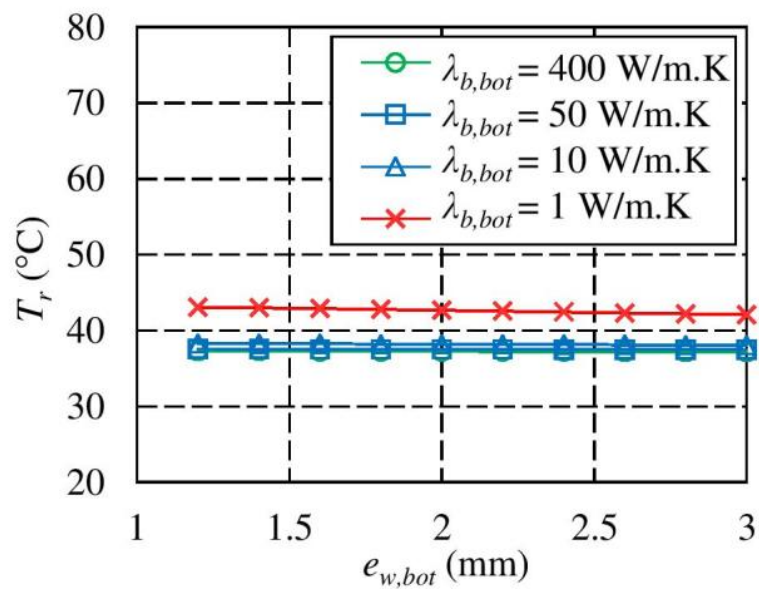

Fig. 4: Effect of the bottom layer properties on the temperature reservoir It is interesting to note that, even if increasing the porosity decreases the effective thermal conductivity according to Eq. (2) (which must be avoided in the case of the bottom layer), it increases the permeability $K$, according to the Blake-Kozeny equation (Carman, 1956): 


$$
K=r_{p}^{2} \frac{\varepsilon^{3}}{30,5(1-\varepsilon)^{2}}
$$

and thus, it decreases the pressure losses as well. Yet, the model results show that the top and bottom layer porosities have a very little influence on the reservoir temperature when ranging between 20 and $70 \%$ (Fig. 5). That is why it is interesting to keep the porosities at a high level since it reduces the pressure losses.

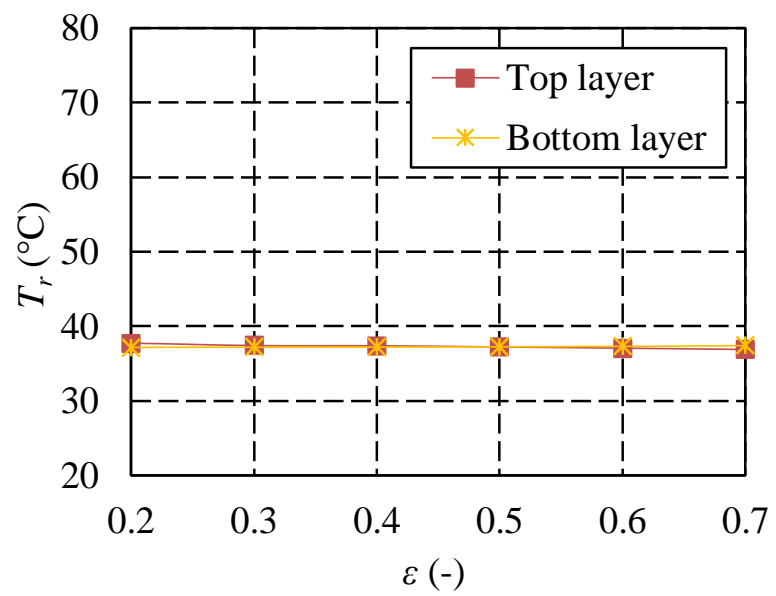

Fig. 5: Effect of the bottom and top layer porosities on the reservoir temperature

Finally, the influence of the top layer pore radius is studied. This pore radius must remain under a certain value to avoid the formation of vapour bubble at the interface between the two layers which can lead to the wick depriming. If this phenomenon occurs, the boiling limit is reached. This risk can be avoided if the following condition is satisfied:

$$
P_{l, \text { cont }}>P_{\text {sat }, v}\left(T_{\text {cont }}\right)-\frac{2 \sigma}{r_{p, t o p}}
$$

where $P_{l, c o n t}$ is the liquid pressure and $P_{s a t, v}$ is the vapour saturation pressure, at the contact between the bottom and the top layers. This condition ensures that the liquid is always in a subcooled state considering a curved interface equals to $r_{p \text {,top. }}$. It underestimates the real value of the boiling limit but ensures a good operation of the LHP. In order to determine the boiling limit, the input heat load is increased in the model until the condition expressed by Eq. (4) is not satisfied anymore. In Fig. 6, the boiling limit is plotted as a function of the top layer pore radius for three different top layer thicknesses. It is clear that the larger the pore radius, the lower the boiling limit. This was expected according to the previous equation. Indeed, a larger top pore radius leads to a smaller difference 
between the saturation pressures of the liquid and the vapour. This difference is easily overcome by the pressure losses inside the wick. For the same reason, a thicker wick decreases the boiling limit as it increases the pressure losses through the top layer and thus, it decreases the liquid pressure at the contact between the two layers. But, as it can be observed in Fig. 6, the effect of the wick thickness is small. In conclusion, a top layer pore radius of $15 \mu \mathrm{m}$ seems to be a good trade-off to ensure a sufficiently high boiling limit of $125 \mathrm{~W}$, corresponding to a heat flux density of about $10 \mathrm{~W} / \mathrm{cm}^{2}$ for a flat disk-shaped evaporator having a diameter of $40 \mathrm{~mm}$.

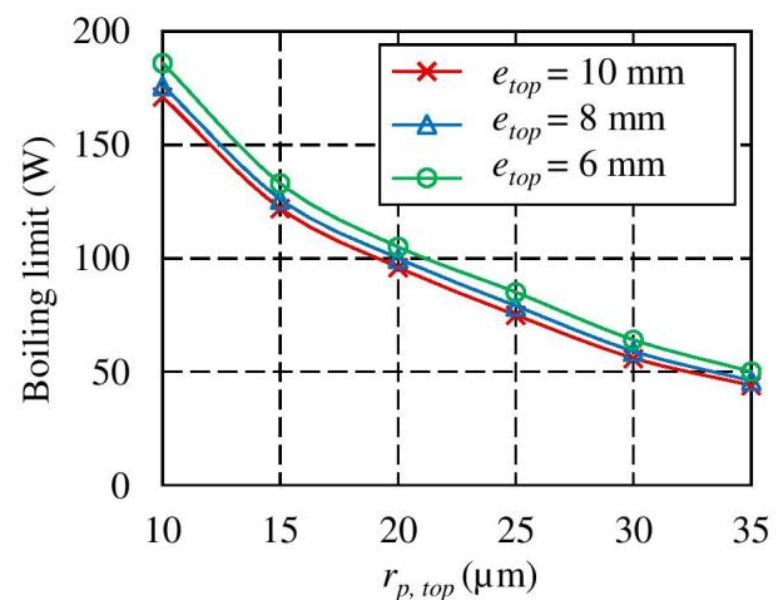

Fig. 6: Influence of the top layer thickness and pore radius on the boiling limit

\subsection{Hydrodynamic behaviour considerations}

In a LHP placed at horizontal position, a fluid set in motion by the capillary pumping pressure is submitted to pressure losses as it circulates around the loop. At each time, the pressure losses must be overcame by the maximum capillary pumping pressure $\Delta P_{c a p, \max }$, respecting the following condition:

$$
\Delta P_{\text {cap }, \max }>\Delta P_{g r}+\Delta P_{V}+\Delta P_{c}+\Delta P_{L}+\Delta P_{w}
$$

where $\Delta P_{g r}$ is the pressure losses in the vapour grooves, $\Delta P_{V}$ in the vapour line, $\Delta P_{c}$ in the condenser, $\Delta P_{L}$ in the liquid line and $\Delta P_{w}$ in the wick. $\Delta P_{w}$ is expressed as follows according to Darcy's law (1856):

$$
\Delta P_{w}=\frac{u_{l} e_{w} \mu_{l}}{K}
$$

where $u_{l}$ is the liquid velocity through the wick, $e_{w}$ is the wick thickness, and $\mu_{l}$ is the fluid viscosity. 
The bottom layer radius has a strong influence on the capillary limit. Indeed, a small pore radius increases the maximum capillary pumping pressure according to the following relationship:

$$
\Delta P_{c a p, \max }=\frac{2 \sigma \cos \theta}{r_{p, b o t}}
$$

where $\sigma$ is the working fluid surface tension, $\theta$ the contact angle between the fluid and the pore wall and $r_{p, b o t}$ the bottom layer pore radius. However, a small pore radius decreases the permeability according to Eq. (3) and thus the capillary limit. That explains why a trade-off exists in the selection of a pore radius value. The capillary limit is determined with the same method as the boiling limit. The heat load is increased step by step in the model while the condition expressed by Eq. (5) is satisfied. Fig. 7 shows that the optimum pore radius is located between 0.5 and $0.8 \mu \mathrm{m}$. However, it seems reasonable to target a larger pore radius for two reasons. The first one is that this capillary limit is determined in the case of a perfectly pure fluid. However, if some impurities are present in the fluid, the small pores could be blocked. The second reason is that the capillary limit is determined in the case of an isotropic porous medium. However, the real porous media have a pore radius distribution which depends on the quality of the metallic powder and on the sintering process. The influence of a variation of the pore size, even a small one, could dramatically decrease the capillary limit under the optimum value. At the contrary, a variation of several microns of the pore size has a small influence when the pore size is beyond the optimum. That is why, a target value of $10 \mu \mathrm{m}$ is chosen in the present study. Indeed, it enables to evacuate more than $10 \mathrm{~W} / \mathrm{cm}^{2}(125 \mathrm{~W}$ for an evaporator having a diameter of $40 \mathrm{~mm}$ ) without risks.

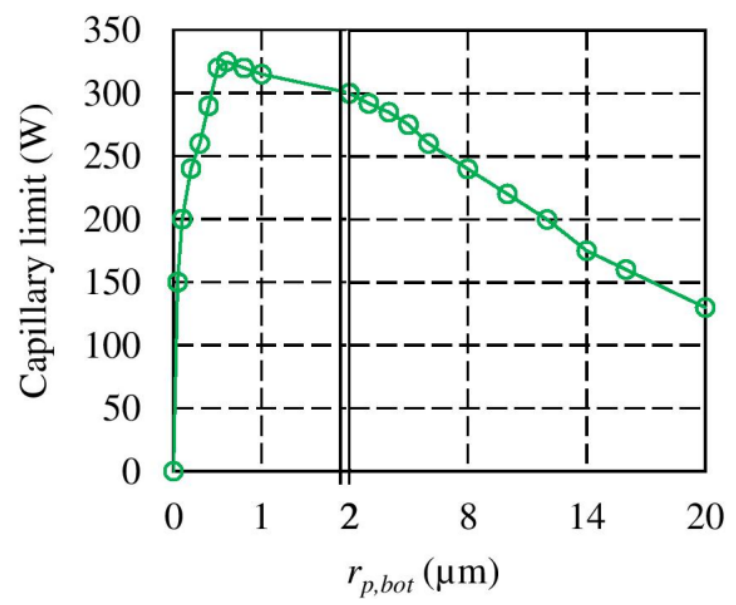

Fig. 7: Capillary limit as a function of the bottom layer pore radius 
The influence of the permeability is also studied. Increasing the wick permeability decreases the pressure losses and increases the capillary limit (Eq. (5) and Eq. (6)). However, Fig. 8 shows that beyond a threshold (around $2.10^{-14} \mathrm{~m}^{2}$ for the bottom layer and $3.10^{-14} \mathrm{~m}^{2}$ for the top layer), increasing the permeability is no longer efficient. This highlights the fact that the pressure losses through the wick are dominant if the permeability is low but become negligible compared to the other pressure losses around the loop beyond the threshold. It means that if the permeability of the layers is high enough, it is not necessary to increase it.

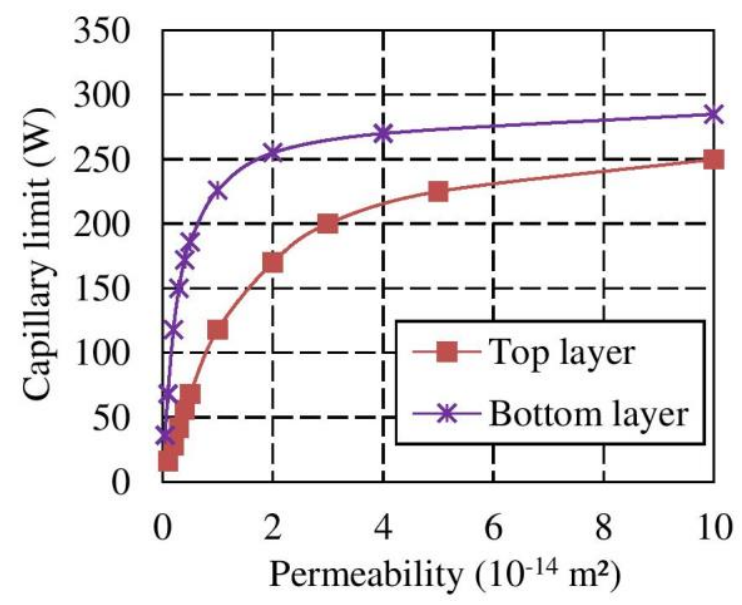

Fig. 8: Capillary limit as a function of the top and bottom layer permeabilities

\subsection{Sizing summary}

Table 3 sums up all the results obtained during the sizing procedure and details the targets in terms of bi-layer wick characteristics. The results of the parametric analysis shows that there is no strict optimum for the different parameters, but rather a trade-off between different considerations (thermal and hydrodynamic performance, mechanical strength, dimensions, etc). It is important to note that these results are only valid in the case of a specific LHP operating with water and having the characteristics described in Table 2. The main result is that there is no optimum set of parameters when modelling a bi-layer wick. Contrarily to a monolayer wick, with a bi-layer one, it is possible to simultaneously strongly decrease the heat leaks and increase the latent heat transfer.

Table 3: targets for the wick characteristics

\begin{tabular}{|c|c|c|c|c|c|}
\hline Layer & $\boldsymbol{e}$ & $\boldsymbol{\varepsilon}$ & $\boldsymbol{K}$ & $\boldsymbol{r}_{\boldsymbol{p}}$ & $\boldsymbol{\lambda}$ \\
\hline Top & $10 \mathrm{~mm}$ & $\begin{array}{c}\text { No influence in the } \\
\text { ranging } 0.2-0.7\end{array}$ & $>3.10^{-14} \mathrm{~m}^{2}$ & $15 \mu \mathrm{m}$ & $<0.1 \mathrm{~W} / \mathrm{m} . \mathrm{K}$ \\
\hline Bottom & $\begin{array}{c}\text { As low as possible while } \\
\text { ensuring a sufficient } \\
\text { mechanical strength }\end{array}$ & $\begin{array}{c}\text { No influence in the } \\
\text { ranging } 0.2-0.7\end{array}$ & $>2.10^{-14} \mathrm{~m}^{2}$ & $10 \mu \mathrm{m}$ & $>10 \mathrm{~W} / \mathrm{m} . \mathrm{K}$ \\
\hline
\end{tabular}




\section{WICK MANUFACTURING}

In the present work, the objective is to obtain a sample having characteristics similar to the optimum ones determined in the previous section. This section presents the manufacturing procedure of the bottom layer only. Indeed, the most important characteristics to determine are the bottom layer ones, since it is the layer where the latent heat transfer takes place. Several porous structures are manufactured in order to understand the effect of the manufacturing process parameters on the wick characteristics.

A classical method to obtain a porous medium is the partial sintering of powders. It consists in heating a powder packing below its melting temperature in order to create bridges between the particles without filling the pores between them. The bridges are created by the diffusion of the atoms from one particle to another. Copper is chosen in order to manufacture the bottom layer, in order to have a high thermal conductivity. According to Chi (1976), the pore radius depends on the powder diameter and can be estimated as follows:

$$
r_{p}=0.41 r_{s}
$$

Where $r_{s}$ is the powder radius. The copper particles used in the present study have a dendritic structure and the following size:

- $\quad 1^{\text {st }}$ decile: $9.1 \mu \mathrm{m}$

- Median diameter: $28.2 \mu \mathrm{m}$

- $9^{\text {th }}$ decile: $59.7 \mu \mathrm{m}$

It corresponds to an expected pore radius of about $6 \mu \mathrm{m}$. It is important to note that this estimation cannot be considered as accurate since the Chi equation (8) is derived for a powder of spheres and not a powder of dendritic particles. However, it is a good indicator to choose the appropriate powder for a specific application.

The sintering process is divided in several steps. Firstly, the powder is mixed with a binder made of polyvinyl alcohol (PVA). The PVA helps the wick to keep its shape before the sintering. The proportion of PVA in the mixture is kept constant. Indeed, a lower ratio would prevent the PVA to keep its binding role and a higher ratio would induce a too long debinding stage. Then, the mixture is pressed in a matrix in order to obtain its shape. The matrix gives a flat-disk shape to the wick with a diameter of $40 \mathrm{~mm}$. The wick is then sintered in an oven at atmospheric pressure. The temperature increases at $1 \mathrm{~K} / \mathrm{min}$ up to $600{ }^{\circ} \mathrm{C}$ (Fig. 9). The dwell time lies between $5 \mathrm{~h}$ and $10 \mathrm{~h}$ long in order to 
remove all the PVA required to obtain a sufficient mechanical strength before the sintering. Then, the temperature increases at $5 \mathrm{~K} / \mathrm{min}$ up to the desired sintering temperature which is one of the parameters. The other parameters of the sintering process are the pressure applied on the sample before the sintering, the copper mass, and the sintering time.

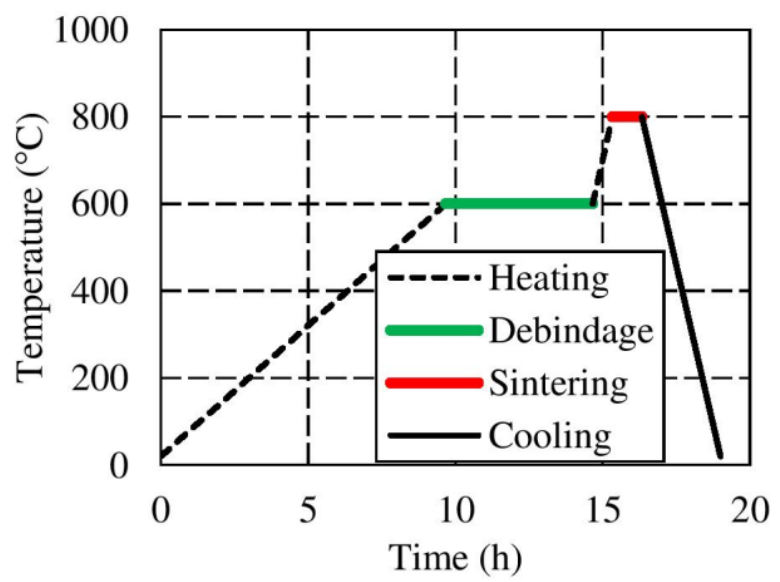

Fig. 9: Temperature profile of the oven during the sintering

Fig. 10 shows a picture of the final product. This wick can directly be used as a bottom layer.

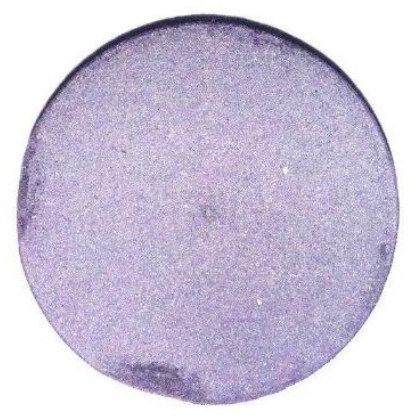

Fig. 10: Picture of the bottom layer

\section{WICK CHARACTERIZATION}

Various methods can be used in order to determine the characteristics of a sample, and particularly its permeability, its porosity and its pore size. These methods are shortly described in the following sections and the results are then detailed and discussed.

\subsection{Characterization by optic microscopy and by tomography}

The pore radius can be determined by X-ray computed tomography (XRCT). The XRCT is a qualitative method that uses X-rays to scan the porous structure in its core and enables reconstituting a 3D image of the sample. Thereby, it is possible to see if the pores are interconnected or not. An example of a picture obtained by tomography is presented in 
Fig. 11 (left). It can be noted that the minimum resolution of such a device $(0.7 \mu \mathrm{m}$ per pixel) is not high enough to detect the small pores in the manufactured samples. However, it is an interesting method to detect large defaults inside the porous wick. Indeed, these defaults cannot be observed with another method as it is the only one that is able to give a view of the inner structure of the porous wick. The pore radius can also be determined by optical microscopy. It gives a large view of the porous sample surface (Fig. 11 right). It is possible to see several small pores as well as some large pores. With both methods, many pore radii are directly measured on the picture obtained and the average pore radius is calculated and called $r_{p, m i c}$. Note that the accuracy of this estimation method depends on the picture definition, the position of the picture on the wick area and the number of pores taken into account. Moreover, the pore are not exactly circular what creates an additional uncertainty. Thus, the uncertainty of these methods is large, but it gives a good order of magnitude of the apparent pore radius.

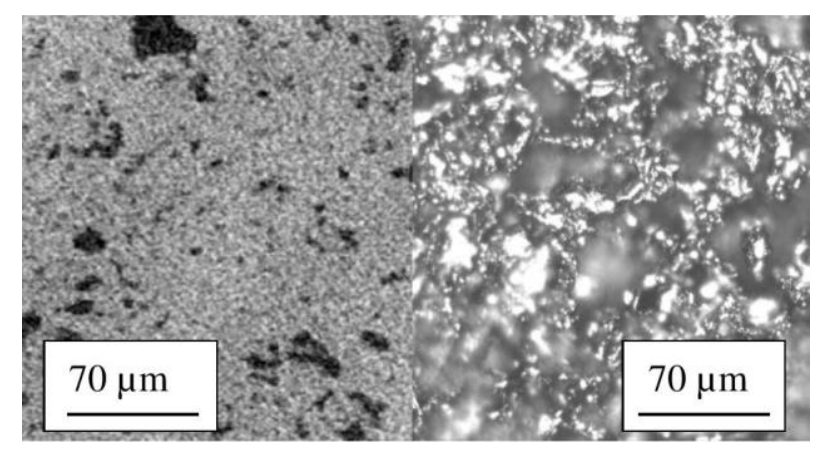

Fig. 11 : Tomography (left) and view by microscopy (right) of a porous sample

\subsection{Capillary pressure test}

An experimental test bench is designed in order to determine the effective pore radius $r_{p, e f f}$. The set-up is composed of a main tube located inside a test tube (Fig. 12 left) and linked to a support in order to let both extremities free. It uses a method similar to the rate-of-rise test proposed by Holley and Faghri (2006). The porous medium is put at the top of the test tube and both tubes are filled with water. The water level in the main tube is then decreased step by step, each step corresponding to $10 \mathrm{~cm}$. The water remains inside the porous medium by capillarity and a hydrostatic pressure is generated between the test tube and the main tube. The hydrostatic pressure is determined by measuring the height of water in the main tube. When the liquid column falls, the maximum capillary pressure that the wick can sustain is reached and $r_{p, \text { eff }}$ is deduced from Eq.(9):

$$
r_{p, e f f}=\frac{2 \sigma}{\Delta P_{c a p, \max }}
$$


With:

$$
\Delta P_{\text {cap }, \max }=\rho_{l} g h_{c a p}
$$

where $\rho_{l}$ is the liquid density and $h_{\text {cap }}$ the maximum height of the water before the depriming of the wick. As the test tube height is $1.8 \mathrm{~m}$, it is not possible to determine effective pore radius smaller than $8 \mu \mathrm{m}$ with pure water $\left(\sigma=72.10^{-3} \mathrm{~N} / \mathrm{m}\right)$. The effective pore radius is determined with an uncertainty ranging from $0.5 \mu \mathrm{m}$ to $3 \mu \mathrm{m}$ depending on the maximum height of the water before the depriming of the wick.

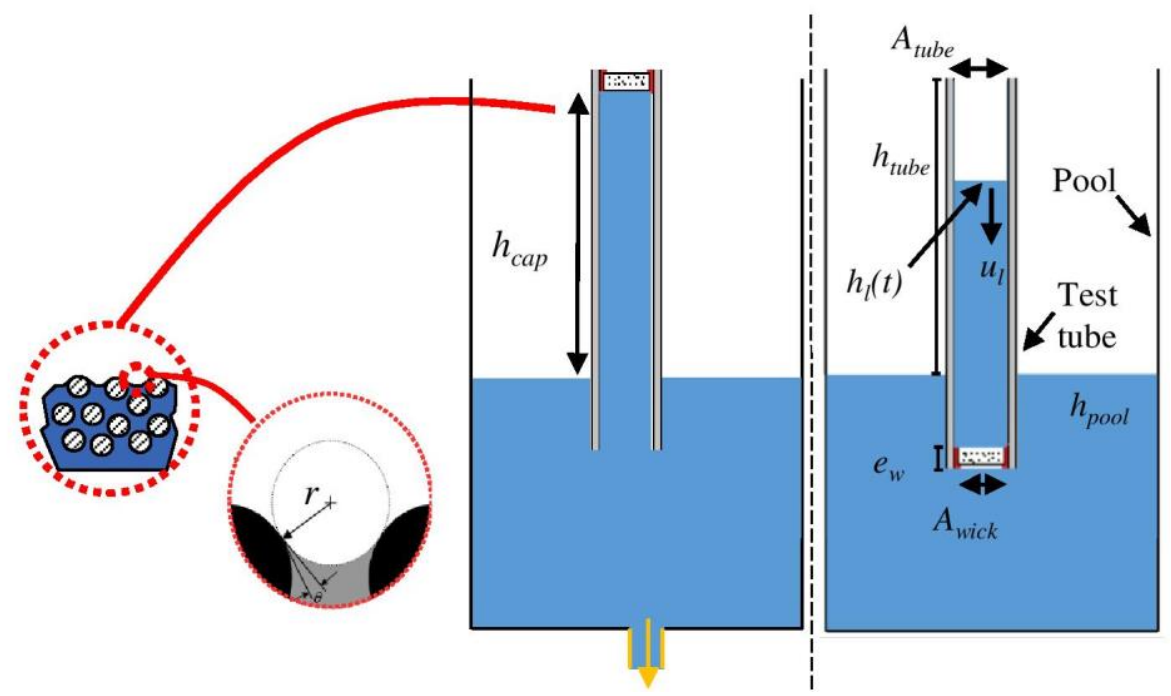

Fig. 12: Schematic of the experimental bench in the two different configurations: pore radius measurement (left) and permeability measurement (right)

\subsection{Permeability measurement by direct method}

The permeability can be directly measured with the same test bench following the method described by Deng et al. (2013). The sample is set at the bottom of the test tube which is filled of liquid (Fig. 12 right). The liquid goes through the porous medium. The determination of the permeability is based on the following expression derived from the Darcy's law (Eq. (6)):

$$
u(t)=\frac{K \Delta P_{w}(t)}{\mu_{l} e_{w}}
$$

Where $u$ is the Darcy's fluid velocity through the wick, $e_{w}$ the wick thickness, $\mu_{l}$ the dynamic viscosity of the liquid and $\Delta P_{w}$ the pressure difference between the top and the bottom of the wick. The singular 
pressure losses at the entrance and exit of the wick are neglected as well as the regular pressure losses along the test tube. $\Delta P_{w}$ is also equal to the hydrostatic pressure of the liquid column:

$$
\Delta P_{w}(t)=\rho_{l} g h_{l}(t)
$$

where $h_{l}$ is the difference between $h_{\text {pool }}$, the height of the water free level in the pool and $h_{\text {tube }}$, the height of the water free level in the test tube. During the experiments, the water free levels are recorded as a function of the time. It enables to determine the Darcy's liquid velocity through the wick by mean of a mass balance:

$$
u_{l}(t)=\frac{A_{\text {tube }}}{A_{w}} \frac{d h_{l}(t)}{d t}
$$

where $A_{\text {tube }}$ is the tube cross sectional area and $A_{w}$ is the wick cross sectional area. The evolution of the Darcy's velocity as a function of $\Delta P_{w}$ is plotted in Fig. 13. A linear regression can be found between the two parameters. It means that the term $K /\left(e_{w} \cdot \mu_{l}\right)$ is a constant and that the Darcy's law is verified. Since the thickness and the fluid viscosity are constant, it is possible to determine the permeability which is equal to $1.7710^{-13} \mathrm{~m}^{2}$ in the present case. This phenomenon being slow, the measure of the water free levels is rather accurate. The main uncertainty corresponds to the variation of the water viscosity due to the ambient temperature fluctuation. As a consequence, the uncertainty of the permeability is lower than $2 \%$.

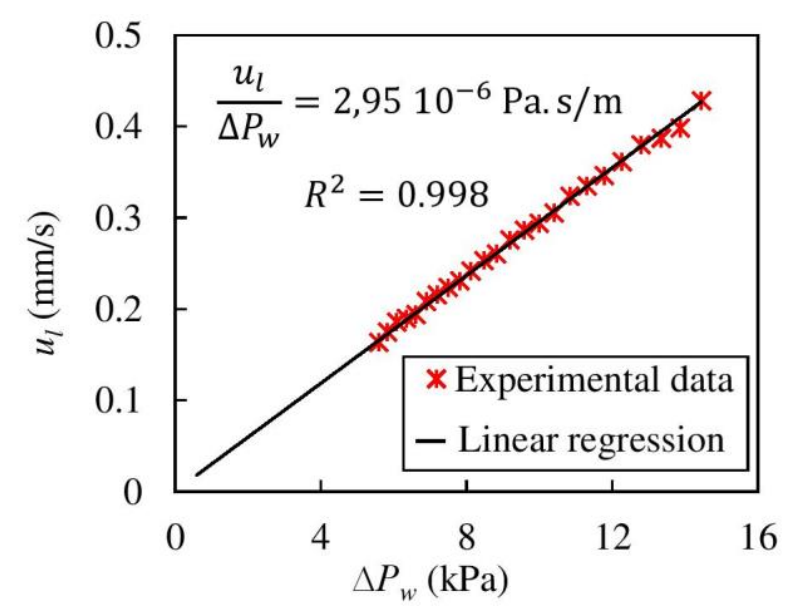

Fig. 13: Darcy's velocity as a function of the pressure difference across the wick

\subsection{Porosity measurement by direct method}


The porosity can be experimentally determined by means of a weighing scale. If the wick mass $m_{w}$, the wick volume $V_{w}$ and the bulk material density $\rho_{b}$ are known, the porosity $\varepsilon$ can be determined using Eq. (14):

$$
\varepsilon=1-\frac{m_{w}}{\rho_{b} V_{w}}
$$

It represents the mass of the pores if they were filled with material, divided by the theoretical mass of a sample having the same volume and made of the same material but without any pores. The mass is known with a large accuracy $(0.01 \mathrm{~g})$ whereas the measurement of the volume is less accurate. Indeed, after the sintering, the porous wick is not perfectly disk-shaped. The uncertainty of the wick diameter and thickness is estimated to about $200 \mu \mathrm{m}$. It leads to an uncertainty of the porosity of about $4 \%$.

\subsection{Effect of the sintering parameters on the characteristics}

The main sintering parameters are the pressure applied on the sample, the mass of sintered powder, the sintering temperature and the sintering time. In order to observe the influence of each of these parameters, as well as the possible interactions between them, the two-level fractional factorial design described by Montgomery (2008) is used. This method limits the number of sample to manufacture by avoiding trying all possible combinations of the sintering parameters. The maximum and minimum values of each of them are detailed in Table 4. Table 5 provides the eight sets of parameters tested in the present study as well as the characteristics determined by means of the different methods for the eight manufactured samples.

Table 4: Sintering parameters

\begin{tabular}{|c|c|c|c|c|}
\hline Parameter & Press. & Mass & Temp. & Time \\
\hline Max (+) & $34.5 \mathrm{MPa}$ & $25 \mathrm{~g}$ & $900{ }^{\circ} \mathrm{C}$ & $2 \mathrm{~h}$ \\
\hline Min (-) & $7 \mathrm{Mpa}$ & $10 \mathrm{~g}$ & $800{ }^{\circ} \mathrm{C}$ & $0.5 \mathrm{~h}$ \\
\hline
\end{tabular}

Table 5: Experiment design and characterization results

\begin{tabular}{|c|c|c|c|c|c|c|c|c|}
\hline & \multicolumn{4}{|c|}{ Sintering parameters } & \multicolumn{4}{c|}{ Experimental characteristics } \\
\hline Sample & Press & Mass & Temp. & Time & $\varepsilon(\%)$ & $\boldsymbol{K}\left(\mathbf{1 0}^{-14} \mathbf{m}^{2}\right)$ & $\boldsymbol{r}_{p, \text { eff }}(\boldsymbol{\mu m})$ & $\boldsymbol{r}_{p, \text { mic }}(\boldsymbol{\mu m})$ \\
\hline 1 & - & - & - & - & 44 & 12 & 16.5 & 12 \\
\hline 2 & + & - & - & + & 23 & 0.18 & $<8$ & 7.0 \\
\hline
\end{tabular}




\begin{tabular}{|c|c|c|c|c|c|c|c|c|}
\hline 3 & - & + & - & + & 41 & 10 & 29.0 & 10 \\
\hline 4 & + & + & - & - & 23 & 0.6 & $<8$ & 8.1 \\
\hline 5 & - & - & + & + & 37 & 11 & 16.3 & 6.6 \\
\hline 6 & + & - & + & - & 28 & 0.52 & 14.7 & 9.5 \\
\hline 7 & - & + & + & - & 43 & 18 & 18.3 & 8.6 \\
\hline 8 & + & + & + & + & 22 & 0.56 & 18.0 & 8.9 \\
\hline
\end{tabular}

Firstly, it is possible to observe that samples 1,3,5 and 7 corresponds very well to the target characteristics detailed in the section 2 of this paper. Indeed, their permeability is higher than the bottom layer threshold $\left(2.10^{-14} \mathrm{~m}^{2}\right)$ and their pore radius corresponds to a capillary limit lying between 150 and $250 \mathrm{~W}$ (Fig. 6) i.e. to heat flux densities ranging between 12 and $20 \mathrm{~W} / \mathrm{cm}^{2}$ ). Finally, since they are made of copper, their thermal conductivity is far above $10 \mathrm{~W} / \mathrm{m} . \mathrm{K}$ (See Table 3: targets for the wick characteristics).

Secondly, it is possible to see that, for a same sample, the pore radius obtained by microscopy and the effective pore radius can be different. When the medium pore radius observed by optic microscopy is smaller than the effective pore radius, it means that there is a large interconnection pore which goes entirely through the wick and prevents a high capillary force. In the opposite case, it means that the porosity is more isotropic. Moreover, the contact angle has an influence when measuring the effective pore radius while it has no influence when observing the sample surface by microscopy. The pore radii observed by microscopy lie between $6.6 \mu \mathrm{m}$ and $12 \mu \mathrm{m}$. These values are slightly above the pore radius predicted by the Chi correlation which is $6 \mu \mathrm{m}$ (Eq. (8)). The observed difference is satisfying since this correlation was developed for spherical powder particles, while the powder used in the present study is dendritic.

Finally, these results enables to know the influent sintering parameters. Table 6 shows the influence of each parameter on the porosity, on the permeability and on the two pore radii obtained. Montgomery (2008) described the method to determine the influence of each parameter: each number of this table represents the mean difference of a given characteristic for a given parameter at its high and low levels, respectively. For example, the four samples obtained with the high sintering pressure have a permeability which is lower by $12 \times 10^{-14} \mathrm{~m}^{2}$ compared to the four samples obtained with a low pressure. 
The pressure has a great influence on the porosity. A possible reason is the large range of pressure investigated, which varies by a factor five. Moreover, under a high pressure, the copper particles which have a dendritic structure can be interleaved or even broken. The pressure should not be so influent if the particles are spherical. The sintering time is also influent but to a less extent. The temperature has a small influence but it is important to keep in mind that the investigated temperature range was narrow ( 800 to $900{ }^{\circ} \mathrm{C}$ ). The results would probably be different with a larger range. The sample mass and so the thickness have very few influence on the wick characteristics as well as the sintering temperature. Finally, even if its influence is low, an increasing sintering time decreases the porosity and $r_{p \text {, mic }}$, which is in good accordance with previous studies (Albertin et al., 2012; Samanta et al., 2013).

Table 6 also shows the influence of the interactions between the sintering parameters on the wick characteristics. A strong interaction between two parameters means that one of the parameter is very influent if the other parameter has a small value but not influent is the other parameter has a high value, or the contrary. In this study, no strong interaction is found. Indeed, since there is mainly one influent parameter, it is not possible to have strong interactions between the parameters. If a strong value were found in this table it would have meant that a parameter has a lot of influence especially for a certain value of the second parameter of the given couple. This doesn't seem to be the case for the sintering process used in the present study.

Table 6: Influence of the sintering parameters on the wick characteristics

\begin{tabular}{|c|c|c|c|c|}
\hline Parameter & $\varepsilon(\%)$ & $K\left(\mathbf{1 0}^{-14} \mathbf{m}^{2}\right)$ & $\begin{array}{c}\boldsymbol{r}_{p, \text { eff }} \\
(\boldsymbol{\mu m})\end{array}$ & $\begin{array}{c}\boldsymbol{r}_{\boldsymbol{p}, \boldsymbol{m i c}} \\
(\boldsymbol{\mu} \mathbf{m})\end{array}$ \\
\hline Pressure & -16 & -12 & -7.9 & -0.9 \\
\hline Mass & -1.0 & 1.5 & 4.5 & 1.3 \\
\hline Temperature & 0.2 & 1.7 & 1.5 & -0.7 \\
\hline Time & -2.6 & -2.4 & 3.5 & -0.4 \\
\hline $\begin{array}{c}\text { Individual influence } \\
\text { Temp./Time }\end{array}$ & -2.2 & -1.3 & -2.8 & -0.3 \\
\hline $\begin{array}{c}\text { Press./Temp. } \\
\text { Mass/Time }\end{array}$ & 2.6 & -1.5 & 6.9 & 1.6 \\
\hline $\begin{array}{c}\text { Press./Time } \\
\text { Mass/Temp. }\end{array}$ & -0.4 & 2.2 & -1.8 & 0.4 \\
\hline
\end{tabular}




\section{CONCLUSIONS}

In the present work, a numerical tool was developed in order to size a bi-layer wick for LHP. By means of this model, the optimum wick properties can thus be determined for a specific LHP geometry in given operating conditions. Some porous structures were manufactured with the aim to get close to the targeted geometrical characteristics of the layer in contact with the heated wall. Indeed, this layer has a large influence on the latent heat transfer while the other has just a role of thermal barrier. A characterization of the hydrodynamic behaviour of the sample was done using various methods in order to determine the porous structure characteristics. Satisfying characteristics and mainly pore radius, thermal conductivity and permeability were obtained. Moreover, a two-level partial fractional design were used in order to understand the sintering process thanks to the characterization. The strong influence of the pressure applied on the porous structure was highlighted compared to the other sintering parameters. Thereby, the method to manufacture the bottom layer were optimized.

They are many perspectives of this work. New types of wick can manufactured with other materials. The objective is to manage a process enabling to manufacture the top layer. Then, a double-layer wick has still to be manufactured. The objective is to avoid a discontinuity at the contact between the two layers and thus, to limit the boiling risk at this interface. The thermal behaviour of a wick is really important to be characterized and a dedicated experimental set up should be created and dedicated to this task. Then, a model should be developed in order to predict the final wick characteristics as a function of the manufacturing parameters. The objective is to develop a tool enabling to manage perfectly the manufacturing process depending on the targeted wick. Finally, the optimized wick should be tested in a real LHP in order to see if the thermal performance are improved as much as predicted by the model.

\section{ACKNOWLEDGEMENT}

The Carnot Institute Ingenierie@ Lyon is gratefully acknowledged for its financial support.

\section{NOMENCLATURE}

$A \quad$ : Cross sectional area $\left(\mathrm{m}^{2}\right)$

$a_{e v} \quad:$ Accommodation coefficient (-)

$c_{p} \quad:$ Specific heat $(\mathrm{J} / \mathrm{kg})$ 


\begin{tabular}{|c|c|}
\hline$D$ & : Diameter $(\mathrm{m})$ \\
\hline$e$ & : Thickness (m) \\
\hline$g$ & : Gravity $\left(\mathrm{m} / \mathrm{s}^{2}\right)$ \\
\hline$H$ & : Heat transfer coefficient $\left(\mathrm{W} / \mathrm{m}^{2} . \mathrm{K}\right)$ \\
\hline$h$ & : Height (m) \\
\hline$h_{l v}$ & : Latent heat of vaporization $(\mathrm{J} / \mathrm{kg})$ \\
\hline$K$ & : Permeability $\left(\mathrm{m}^{2}\right)$ \\
\hline$k_{n}$ & : Coefficients \\
\hline$L$ & : Length (m) \\
\hline$m$ & : Mass (kg) \\
\hline$\dot{m}$ & : Mass flow rate $(\mathrm{kg} / \mathrm{s})$ \\
\hline$P$ & : Pressure $(\mathrm{Pa})$ \\
\hline$Q$ & : Heat flux (W) \\
\hline$r$ & : Radius (m) \\
\hline$R$ & : Thermal resistance $(\mathrm{K} / \mathrm{W})$ \\
\hline$T$ & : Temperature (K) \\
\hline$t$ & : Time $(\mathrm{s})$ \\
\hline$u$ & : Velocity $(\mathrm{m} / \mathrm{s})$ \\
\hline$V$ & : Volume $\left(\mathrm{m}^{3}\right)$ \\
\hline & Greek symbols \\
\hline$\Delta$ & : Difference (-) \\
\hline$\varepsilon$ & : Porosity (-) \\
\hline$\lambda$ & : Thermal conductivity $(\mathrm{W} / \mathrm{m} . \mathrm{K})$ \\
\hline$\mu$ & : Dynamic viscosity (Pa.s) \\
\hline$\rho$ & : Density $\left(\mathrm{kg} / \mathrm{m}^{3}\right)$ \\
\hline$\sigma$ & : Surface tension $(\mathrm{N} / \mathrm{m})$ \\
\hline$\theta$ & : Contact angle $\left(^{\circ}\right)$ \\
\hline & Subscripts \\
\hline $\mathrm{amb}$ & : ambient \\
\hline $\mathrm{b}$ & : bulk wick material \\
\hline body & : evaporator body \\
\hline bot & : bottom layer \\
\hline $\mathrm{c}$ & : condenser \\
\hline
\end{tabular}




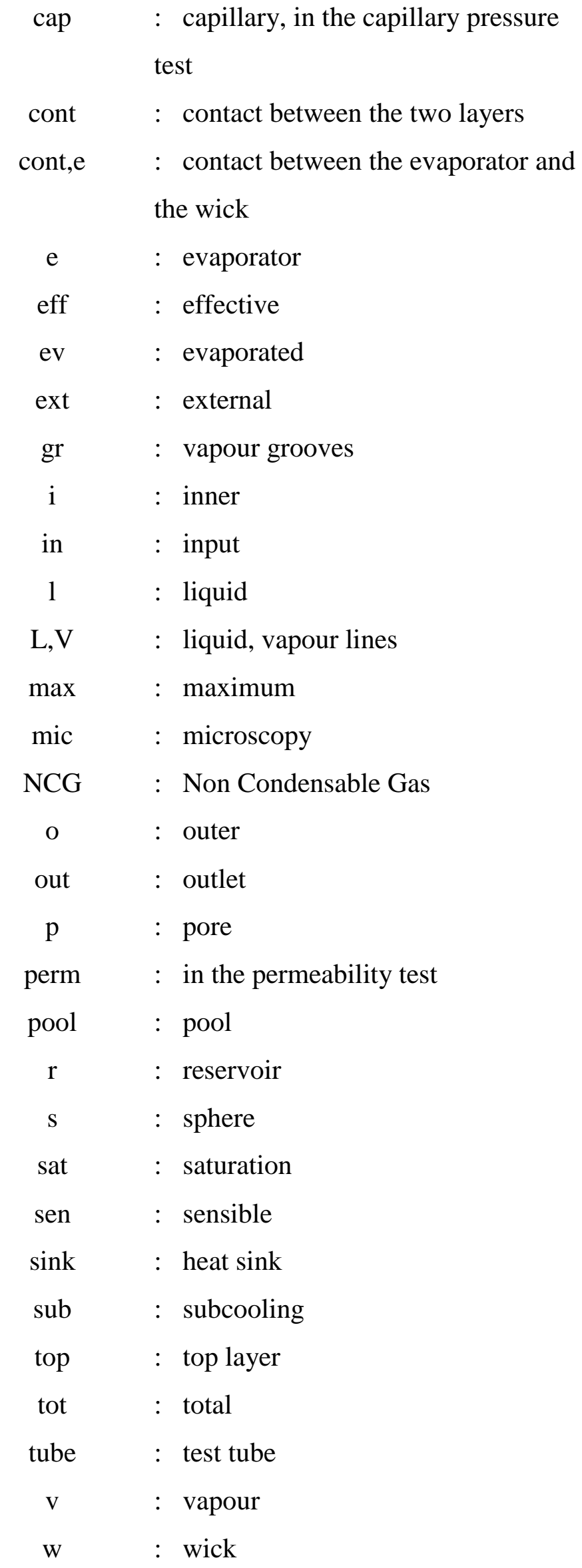




$$
\begin{array}{ll}
\text { wall } & : \text { line wall } \\
\text { we } & : \text { evaporator side of the wick } \\
2 \varphi & : \text { two-phase }
\end{array}
$$

\section{REFERENCES}

Albertin, T., Savard, J.-F., Sartre, V., 2012. Industrial manufacturing of loop heat pipe porous media, in: Proc. 16th International Heat Pipe Conference (16th IHPC). Lyon, France.

Alexander, E.G., 1972. Structure-property relationships in heat pipe wicking materials (Ph. D. Thesis). Departement of Chemical Engineering, North Carolina State University.

Boo, J.H., Chung, W.B., 2005. Experimental Study on the Thermal Performance of a Small-scale Loop Heat Pipe with Polypropylene Wick. J. Mech. Sci. Technol. 19, 1052-1061. doi:10.1007/BF02919189

Cao, Y., Faghri, A., 1994. Analytical solutions of flow and heat transfer in a porous structure with partial heating and evaporation on the upper surface. Int. J. Heat Mass Transf. 37, 1525-1533. doi:10.1016/0017-9310(94)90154-6

Carman, P.C., 1956. Flow of gases through porous media. Academic press.

Chi, S.W., 1976. Heat pipe theory and practice: a sourcebook. Hemisphere Pub. Corp.

Darcy, H., Darcy, H., Darcy, H., 1856. Les fontaines publiques de la ville de Dijon.

Demidov, A.S., Yatsenko, E.S., 1994. Investigation of heat and mass transfer in the evaporation zone of a heat pipe operating by the inverted meniscus' principle. Int. J. Heat Mass Transf. 37, 2155-2163. doi:10.1016/0017-9310(94)90317-4

Deng, D., Liang, D., Tang, Y., Peng, J., Han, X., Pan, M., 2013. Evaluation of capillary performance of sintered porous wicks for loop heat pipe. Exp. Therm. Fluid Sci. 50, 1-9. doi:10.1016/j.expthermflusci.2013.04.014

Holley, B., Faghri, A., 2006. Permeability and effective pore radius measurements for heat pipe and fuel cell applications. Appl. Therm. Eng. 26, 448-462. doi:10.1016/j.applthermaleng.2005.05.023

$\mathrm{Ku}$, J., 1999. Operating Characteristics of Loop Heat Pipes, in: 29th International Conference on Environmental System. pp. 503--519.

Launay, S., Sartre, V., Bonjour, J., 2008. An analytical Model for Characterization of Loop Heat Pipes. J. Thermophys. Heat Transf. 22, 623-631. doi:10.2514/1.37439

Launay, S., Vallée, M., 2011. State-of-the-Art Experimental Studies on Loop Heat Pipes. Front. Heat Pipes 2. doi:10.5098/fhp.v2.1.3003

Maydanik, Y.F., 2005. Loop heat pipes. Appl. Therm. Eng. 25, 635-657. doi:http://dx.doi.org/10.1016/j.applthermaleng.2004.07.010

Montgomery, D.C., 2008. Design and analysis of experiments. John Wiley \& Sons.

Mottet, L., Coquard, T., Prat, M., 2015. Three dimensional liquid and vapour distribution in the wick of capillary evaporators. Int. J. Heat Mass Transf. 83, 636-651. doi:10.1016/j.ijheatmasstransfer.2014.12.048

Samanta, S.K., DAS, P.R., Lohar, A.K., Roy, H., Kumar, S., Chowdhury, A.K., 2013. A novel approach of manufacturing Nickel Wicks for loop heat pipes using Metal Injection Moulding (MIM). Sadhana 38, 281-296. doi:10.1007/s12046-013-0135-3

Santos, P.H.D., Bazzo, E., Becker, S., Kulenovic, R., Mertz, R., 2010. Development of LHPs with ceramic wick. Appl. Therm. Eng. 30, 1784-1789. doi:10.1016/j.applthermaleng.2010.04.010

Siedel, B., Sartre, V., Lefèvre, F., 2015. Complete analytical model of a loop heat pipe with a flat evaporator. Int. J. Therm. Sci. 89, 372-386. doi:10.1016/j.jithermalsci.2014.11.014

Singh, R., Nguyen, T., Mochizuki, M., 2014. Capillary evaporator development and qualification for loop heat pipes. Appl. Therm. Eng. 63, 406-418. doi:10.1016/j.applthermaleng.2013.10.059

Wu, S.-C., Huang, C.-J., Chen, S.-H., Chen, Y.-M., 2013. Manufacturing and testing of the double-layer wick structure in a loop heat pipe. Int. J. Heat Mass Transf. 56, 709-714. doi:10.1016/j.ijheatmasstransfer.2012.09.054

Xu, J., Ji, X., Yang, W., Zhao, Z., 2014. Modulated porous wick evaporator for loop heat pipes: Experiment. Int. J. Heat Mass Transf. 72, 163-176. doi:10.1016/j.ijheatmasstransfer.2014.01.005 
Yeh, C.C., Liu, B.H., Chen, Y.M., 2008. A study of loop heat pipe with biporous wicks. Heat Mass Transf. 44, 1537-1547. doi:10.1007/s00231-008-0387-3

Zhao, T., Liao, Q., 2000. On capillary-driven flow and phase-change heat transfer in a porous structure heated by a finned surface: measurements and modeling. Int. J. Heat Mass Transf. 43, 1141-1155. doi:10.1016/S0017-9310(99)00206-9 